\title{
Transgastric endoscopic gallbladder polypectomy and cholecystolithiasis: A case report
}

\author{
YANG LI $^{*}$ and SHUTANG HAN*

\begin{abstract}
Gastroenterology Endoscopy Center, Affiliated Hospital of Nanjing University of Chinese Medicine,
\end{abstract} \\ Jiangsu Province Hospital of Chinese Medicine, Nanjing, Jiangsu 210029, P.R. China
}

Received June 26, 2019; Accepted October 11, 2019

DOI: $10.3892 /$ etm.2019.8195

\begin{abstract}
As the most common digestive-system disease, cholelithiasis and gallbladder polyps have a high incidence. The most common treatment is laparoscopic cholecystectomy, but there are numerous drawbacks, including stump syndrome. In the present study, a novel treatment, namely transgastric endoscopic gallbladder polypectomy and cholecystolithiasis, was applied. To the best of our knowledge, the present study is the first to report on this application, which can potentially avoid open surgery and associated scars and allows for rapid recovery after surgery, and may therefore be worthy of further development and implementation in clinical practice. It is esteemed that in the future, transgastric endoscopy gallbladder polypectomy and cholecystolithiasis will be considered as a treatment option for certain patients and is subjected to constant improvements.
\end{abstract}

\section{Introduction}

As the most common disease of the digestive system, cholelithiasis and the formation of gallbladder polyps have a high incidence (1). Open cholecystectomy and laparoscopic cholecystectomy are classic methods for the treatment of cholelithiasis and gallbladder polyps and have been widely used in clinical practice. After cholecystectomy, however, certain patients are prone to dyspepsia, reflux esophagitis, gastritis and post-cholecystectomy syndrome, which has raised concern among clinicians (1). Furthermore, studies have

Correspondence to: Dr Yang Li or Professor Shutang Han, Gastroenterology Endoscopy Center, Affiliated Hospital of Nanjing University of Chinese Medicine, Jiangsu Province Hospital of Chinese Medicine, 155 Hanzhong Road, Nanjing, Jiangsu 210029, P.R. China

E-mail: ayly0550@163.com

E-mail: shutanghanhst@163.com

${ }^{*}$ Contributed equally

Abbreviations: EUS, endoscopic ultrasound; NOTES, Natural Orifice Transluminal Endoscopic Surgery

Key words: natural orifice transluminal endoscopic surgery, transgastric, gallbladder polyp, polypectomy, cholecystolithiasis indicated that after cholecystectomy, the incidence of colon cancer, pancreatic cancer, esophageal squamous carcinoma and hepatocellular carcinoma was increased (2-5). However, with increasing knowledge on gallbladder physiology, the significance of the gallbladder is being increasingly recognized. The gallbladder is not only an important component of the human digestive system but also an immune organ: The gallbladder is able to secrete mucus and $\operatorname{IgA}$ antibodies and participate in the construction of the biliary immune defense system $(6,7)$. Therefore, certain clinicians recommend that patients undergo cholecystolithotomy with preservation of the gallbladder, which is able to treat cholelithiasis whilst preserving the function of the gallbladder (8). Furthermore, certain patients refuse to undergo open or laparoscopic cholecystectomy due to this causing surgical scars on the abdominal wall. It is thought that these scars have negative effects on their physical or mental wellbeing (9). With the rapid development of endoscopic technology, cholecystolithotomy with preservation of the gallbladder and no surgical scars is possible.

\section{Case report}

A 45-year-old male patient presented at the Affiliated Hospital of Nanjing Medical University of Traditional Chinese Medicine (Nanjing, China) in May, 2018 and requested the removal of his cholecystic polyps and cholecystolithiasis which had been diagnosed earlier in the outpatient department of the hospital with preservation of the gallbladder. Over the past 2 years, the patient had experienced frequent abdominal pain and fever. Cholecystic polyps and gallstones were observed by pre-operative abdominal ultrasonography (Fig. 1A) and abdominal CT (Fig. 1B). It was estimated that the maximum diameter of cholecystic polyps was $1.2 \mathrm{~cm}$ and that of the biggest stone was $0.8 \mathrm{~cm}$. The pre-operative gallbladder ejection function was deemed good according to gallbladder contraction function test under abdominal ultrasound. Following consultation of relevant literature and discussion with certain endoscopy experts, it was decided that transgastric endoscopic gallbladder polypectomy and cholecystolithiasis was feasible in this patient (10-12). The present study reports on the successful use of flexible transgastric endoscopy in gallbladder polypectomy and treating cholecystolithiasis, which appears to be a promising novel surgical option for patients wishing to retain their gallbladder and 

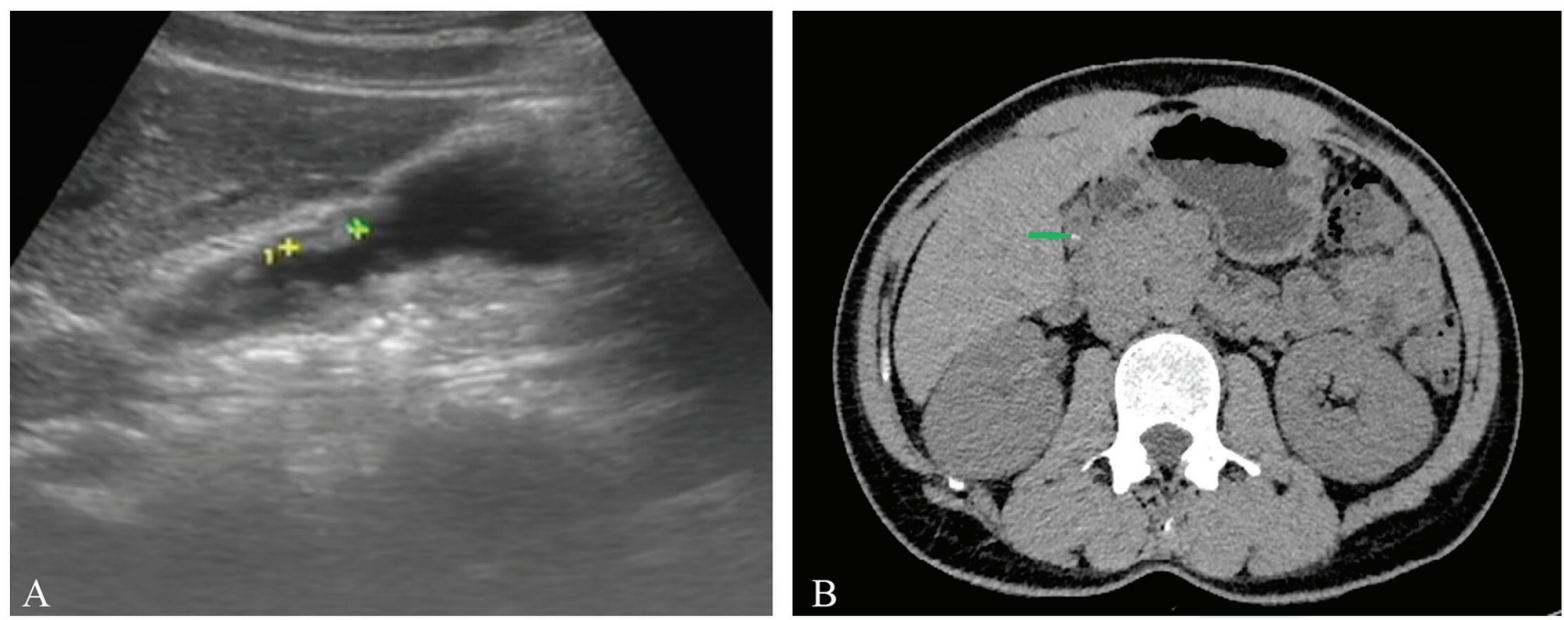

Figure 1. (A) Pre-operative abdominal ultrasonography was performed. The maximum diameter of the cholecystic polyps was $1.2 \mathrm{~cm}$ (indicated between green '+' and yellow '+' symbol). (B) Gallstones were observed by pre-operative abdominal CT (green arrow).

functions without scarring on the abdominal wall. Prior to the operation, written informed consent was obtained from the patient and the operation was approved by written the Ethics Committee of Affiliated Hospital of Nanjing University of Chinese Medicine (Nanjing, China).

A second-generation cephalosporin was prophylactically administered to prevent infection at $30 \mathrm{~min}$ prior to surgery. With the assistance of a transparent cap, an endoscopic full-thickness incision was made on the posterior antrum of the stomach near the small curved side by using a hook knife (Olympus Corp.) and an $\mathrm{IT}_{2}$ knife (Olympus Corp.). After full-thickness dissection, the omentum present in the abdominal cavity was separated layer by layer (Fig. 2A). The gallbladder exhibited mild edema (Fig. 2B). A small incision was opened at the junction of the gallbladder by using the hook knife. With the same tool, the window was extended to allow the endoscope to enter the gallbladder (Fig. 2C). An endoclip with endoloop was used to pull the gallbladder so that the endoscope was able to enter the gallbladder easily. During this procedure, the bile overflowing from the gallbladder into the abdominal cavity was cleaned up. After rinsing the inner wall of the gallbladder with aseptic saline repeatedly, three gallstones were removed (Fig. 2D). The cholecystic polyps were resected from the gallbladder by snare traction and assistance of hot biopsy forceps (Fig. 2E and F). After local hemostasis, the endoscope was retracted from the gallbladder and four endoclips (Micro-Tech) were used to clip the gallbladder incision. The endoscope was withdrawn from the stomach and the incision was closed with endoclips (Micro-Tech) (Fig. 2G). The operation time was $120 \mathrm{~min}$ and no bleeding was observed during surgery. Two sediment-like stones and two larger polyps were successfully removed. The maximum diameter of the extracted gallstones was $1.5 \mathrm{~cm}$ and that of the cholecystic polyps was $0.5 \mathrm{~cm}$ (Fig. $2 \mathrm{H}$ ).

The pathologic diagnosis of the cholecystic polyps was cholesterol polyp (Fig. 2I). The patient recovered without any incident and discharged by the hospital in 2 days. During the three-month follow-up after the endoscopy operation, the patient had no specific discomfort and no gallstones and polyps were observed by transabdominal ultrasound.

\section{Discussion}

With the rapid development of endoscopic technology, novel approaches for treating cholecystic polyps and gallstones are becoming available. Natural Orifice Transluminal Endoscopic Surgery (NOTES) is an improvement in surgical interventions and has been widely used in the clinic. In 2007, Marescaux et al (13) reported on the use of transvaginal endoscopic cholecystectomy for the first time. In 2015, Liu et al (14) performed the first NOTES cholecystolithotomy with gallbladder preservation by flexible endoscopy through a rectal approach. However, due to the presence of intestinal bacteria, it may easily cause contamination through incisions and abdominal infection. In 2018, Schwaitzberg et al (15) reported on transgastric endoscopy cholecystectomy performed in 4 cases. To the best of our knowledge, endoscopic cholecystic polypectomy and cholecystolithiasis in clinical patients has not been previously described. The present study reports on the successful use of endoscopy gallbladder polypectomy and cholecystolithiasis with a transgastric approach by flexible endoscopy, which appears to be a promising novel surgical option for patients wishing to retain their gallbladder and its functions with no scar on the abdominal wall.

In the case of the present study, the patient had suffered from recurrent low fever and abdominal pain for a long time. Gallstones and cholecystic polyps were identified in the pre-operative examination. These are two indications for surgical treatment. Furthermore, the patient did not wish to have any surgical scars on his abdominal wall. Hence, transgastric endoscopic gallbladder polypectomy and cholecystolithiasis was performed. This type of surgery was a huge challenge for endoscopists. Firstly, the snare or forceps polypectomy should completely remove the pathological tissue and avoid gallbladder perforation. This technique was translated from the removal of colon polyps using endoscopy. Submucosal injection in the gallbladder wall and electrocoagulation technology may avoid gallbladder perforation and reduce bleeding (16). Furthermore, the gallbladder and stomach wounds generated by this operation must be treated with caution. In the approach reported in the present study, intensive endoclips and a full set of endoloop 

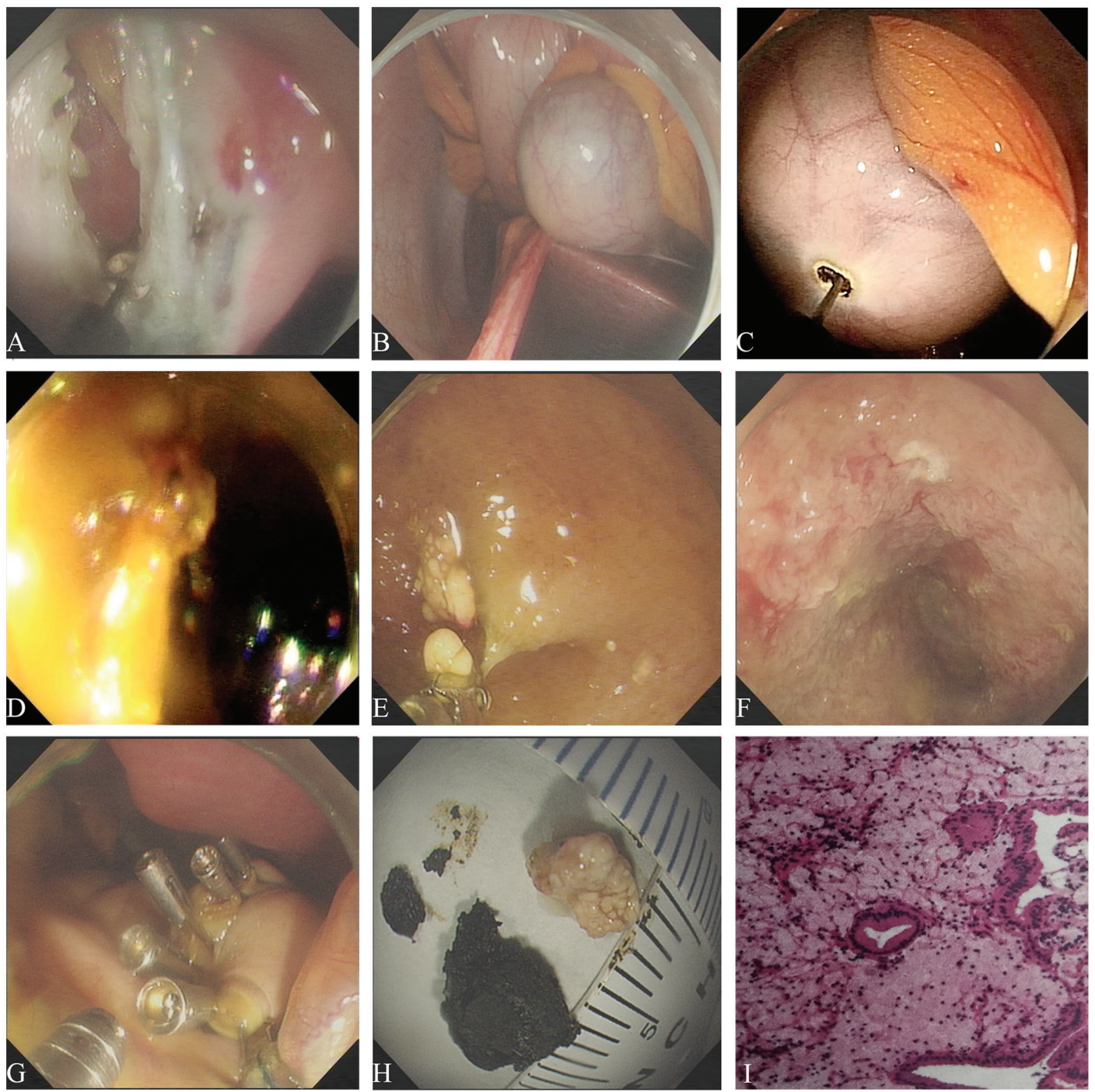

Figure 2. (A) With the assistance of a transparent cap, an endoscopic full-thickness incision was made on the posterior antrum of the stomach near the small curvature by using a hook knife and IT $_{2}$ knife. (B) After the full-thickness dissection, the omentum present in the abdominal cavity was separated layer by layer. (C) By using the same tools, dissection was performed, allowing the endoscope to enter the gallbladder. (D) A 15x15 mm gallstone was pulled out with the endoscope. (E) The polyps were resected from the gallbladder with snare traction assisted by hot biopsy forceps. (F) Gallstones and polyps were cleaned up. (G) After local hemostasis, a total of 5 endoclips (Micro-Tech) were used to clip the gallbladder incision. (H) Images of the bigger gallstones and polyps extracted (1:1). (I) Representative histology image of gallbladder polyps (H\&E staining; magnification, x40); the pathological diagnosis was cholesterol polyp.

successfully solved this problem. In terms of post-operative recovery, these strategies achieved desirable outcomes. Initially, the prevention of abdominal scarring rendered it more feasible for patients to undergo surgery. This type of operation preserved gallbladder function, reduced postoperative complications such as dyspepsia caused by removal of the gallbladder and enhanced postoperative recovery. However, prior to its eventual application in clinical routine, two questions regarding the method require to be addressed, including the possibility of intra-abdominal infection caused by the endoclips which is left in the abdominal cavity following surgery, whereas the other is anastomotic fistula due to poor closure. At our institution, continuous efforts will be made to further develop and eventually include this procedure in clinical routine as soon as possible.

In conclusion, the present study provided the novel technique of transgastric endoscopy gallbladder polypectomy and cholecystolithiasis, an innovative method that should be of interest for interventional endoscopists and may expand the current endoscopic repertoire. With its constant improvement, this method may increasingly become an option for certain patients.

\section{Acknowledgements}

The authors would like to thank Professor Hailin Jin, Professor Jun Xiao, Professor Qide Zhang, Professor Yuhong Zhou, Dr Dapeng Wu, Miss Lu Ze, Miss Wenxia Gu and Miss Jie Su from The Gastroenterology Endoscopy Center, Affiliated Hospital of Nanjing University of Chinese Medicine, Jiangsu Province Hospital of Chinese Medicine (Jiangsu, China). Professor Hailin Jin was the second assistant of this operation. Professor Jun Xiao, Professor Qide Zhang and Professor Yuhong Zhou participated in the formulation of the surgical plan. Dr Dapeng Wu and Miss Jie Su were responsible for the perioperative treatment of this patient. Miss Lu Ze and Miss Wenxia Gu were the surgical nurses of this operation. 


\section{Funding}

The present study was supported by the second batch of scientific research special projects for the construction of the National TCM Clinical Research Base in 2015 (grant no. JDZX2015086), an innovation fund of the Jiangsu Province Hospital of Chinese Medicine (grant no. Y2018CX57) and the Jiangsu Province '333 Project' Training Fund (grant no. BRA2017551).

\section{Availability of data and materials}

All data generated or analyzed during the present study are included in this published article.

\section{Authors' contributions}

YL was the first assistant during the operation and wrote the manuscript. SH was the endoscopy surgeon of this operation and approved the final version of the manuscript. All authors read and approved the final manuscript.

\section{Ethics approval and consent to participate}

Approval was obtained from the Medical Ethics Committee of Affiliated Hospital of Nanjing University of Chinese Medicine (No. 2018NL-094-0; Jiangsu Province Hospital of Chinese Medicine; Nanjing, China).

\section{Patient consent for publication}

The patient provided written informed consent for the publication of data and images.

\section{Competing interests}

The authors declare that they have no competing interests.

\section{References}

1. Saxena P and Khashab MA: New NOTES clinical training and program development. Gastrointest Endosc Clin N Am 26: $385-400,2016$
2. Saliba F, Lakehal M, Pageaux GP, Roche B, Vanlemmens C, Duvoux C, Dumortier J, Salamé E, Calmus Y and Maugendre D; Diapason Study Group: Risk factors for new-onset diabetes mellitus following liver transplantation and impact of hepatitis $\mathrm{C}$ infection: An observational multicenter study. Liver Transpl. 13: 136-144, 2007.

3. Lin G, Zeng Z, Wang X, Wu Z, Wang J, Wang C, Sun Q, Chen Y and Quan H: Cholecystectomy and risk of pancreatic cancer: A meta-analysis of observational studies. Cancer Causes Control 23: 59-67, 2012.

4. Lagergren $\mathbf{J}$ and Mattsson F: Cholecystectomy as a risk factor for oesophageal adenocarcinoma. Br J Surg 98: 1133-1137, 2011.

5. Lagergren J, Mattsson F, El-Serag H and Nordenstedt $\mathrm{H}$ : Increased risk of hepatocellular carcinoma after cholecystectomy. Br J Cancer 105: 154-156, 2011.

6. Maurer KJ, Carey MC and Fox JG: Roles of infection, inflammation, and the immune system in cholesterol gallstone formation. Gastroenterology 136: 425-440, 2009.

7. Martelli MG and Lee JY: Parasitic infection of the gallbladder: Cystoisospora belli infection as a cause of chronic abdominal pain and acalculous cholecystitis. J Miss State Med Assoc 57: 174-176, 2016.

8. Soltes M and Radoňak J: A risk score to predict the difficulty of elective laparoscopic cholecystectomy. Wideochir Inne Tech Maloinwazyjne 9: 608-612, 2014.

9. Ko WJ, Song GW, Sukpyo S, Kim DH, Yoo JH, WonHee K, Kwon C II, Ko KH, Hahm KB, Hong SP, et al: Natural orifice translumenal endoscopic surgery: Current status and future perspective. Gastrointestinal Endoscopy 83: AB647, 2016.

10. Morgan M, Olweny EO and Cadeddu JA: LESS and NOTES instrumentation: Future. Curr Opin Urol 24: 58-65, 2014.

11. Zorron R, Phillips HN, Wynn G, Neto MP, Coelho D and Vassallo RC: 'Down-to-Up' transanal NOTES Total mesorectal excision for rectal cancer: Preliminary series of 9 patients. J Minim Access Surg 10: 144-150, 2014.

12. Bernhardt J, Sasse S, Ludwig K and Meier PN: Update in natural orifice translumenal endoscopic surgery (NOTES). Curr Opin Gastroenterol 33: 346-351, 2017.

13. Marescaux J, Dallemagne B, Perretta S, Wattiez A, Mutter D and Coumaros D: Surgery without scars: Report of transluminal cholecystectomy in a human being. Arch Surg 142: 823-827, 2007.

14. Liu B, Du B and Pan Y: Video of the Month: Transrectal gallbladder-preserving cholecystolithotomy via pure natural orifice transluminal endoscopic surgery: First time in humans. Am J Gastroenterol 110: 1655, 2015.

15. Schwaitzberg SD, Roberts K, Romanelli JR, Desilets DJ, Earle D, Horgan S, Swanstrom L, Hungness E, Soper N, Kochman ML, et al: The NOVEL trial: Natural orifice versus laparoscopic cholecystectomy-a prospective, randomized evaluation. Surg Endosc 32: 2505-2516, 2018.

16. Tang P, Zhang X, Guo H, Kong J, Sun G, HL E and Yang Y: Natural orifice transgastric endoscopic operation for diaphragmatic repair in a dog model. Biomedical Res 28: 1333-1336, 2017.

This work is licensed under a Creative Commons Attribution-NonCommercial-NoDerivatives 4.0 International (CC BY-NC-ND 4.0) License. 